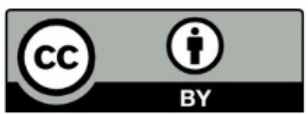

\title{
Estrategias de aprendizaje
}

\author{
Learning strategies
}

Estratégias de aprendizado

\section{ARTÍCULO GENERAL}

Henry Camizán García

https://orcid.org/0000-0002-0350-9078

hcamizan@ucvvirtual.edu.pe

Universidad César Vallejo, Lima - Peru
Benites Seguín, Lucio Alfredo

https://orcid.org/0000-0002-4392-1403

lbenitess@ucvvirtual.edu.pe

Universidad César Vallejo, Lima - Peru
Damián Ponte, Isaías Francisco

https://orcid.org/0000-0002-1005-2109

idamianp@ucvvirtual.edu.pe

Universidad César Vallejo, Lima - Peru

Recibido 08 de Noviembre 2020 | Arbitrado y aceptado 28 de Noviembre 2020 | Publicado en 05 Marzo 2021

\section{RESUMEN}

Al hacer un análisis de la realidad educativa nacional, se tuvo que lo fundamental para los estudiantes debe ser el nivel de comprensión del manejo de información y la utilización de las estrategias de aprendizaje. Es una exigencia social que ellos adquieran - capacidades de aprendizaje que les permita adaptarse mejor al nivel superior y las nuevas demandas laborales. Los estudiantes deben ser capaces de aprender en forma autónoma y autorregulada. Eligen y activan aquellos conocimientos que necesita para responder a las exigencias de la demanda profesional y personal. en función de las condiciones de la situación educativa Las estrategias de aprendizaje se definen en relación con la toma de decisiones En este articulo se ofrecen las pautas básicas, como son las definiciones y marcos conceptuales relacionados a las estrategias de aprendizaje para una mejor comprensión del tema por parte de los docentes en el proceso de enseñanza - aprendizaje. Las estrategias de aprendizaje se utilizan cuando el estudiante da muestras de ajustarse continuamente a los cambios y variaciones que se van produciendo en el transcurso de la actividad siempre con la finalidad última de alcanzar el objetivo perseguido del modo más eficaz que sea posible. De esta forma, el estudiante minimiza el número de errores previos a la solución del problema, asegurando que su respuesta sea la correcta después de un minimo de tentativas. Además, el aprendizaje autónomo es el proceso que le permite al estudiante regular lo que aprende y ser consciente de sus procesos cognitivos y socio afectivos. El esfuerzo pedagógico se centra en la formación de individuos con capacidad de solucionar aspectos especificos de su propio aprendizaje, es decir, orientar al educando a que se cuestione, revise, planifique, controle y evalúe su propia acción de aprender a aprender e imprimir aprendizajes significativos utilizando estrategias adecuadas y motivadoras en su proceso a aprendizaje.

Palabras clave: Estrategias de aprendizaje, aprendizaje en escolares.

\section{ABSTRACT}

When making an analysis of the national educational reality, the fundamental thing for students should be the level of understanding of information management and the use of learning strategies. It is a social requirement that they acquire - learning capacities - that allows them to better adapt to the higher level and the new job demands. Students must be able to learn autonomously and self-regulating. They choose and activate the knowledge they need to meet the demands of professional and personal demand, depending on the conditions of the educational situation. Learning strategies are defined in relation to decision making.

This article offers the basic guidelines, such as definitions and conceptual frameworks related to learning strategies for a better understanding of the subject by teachers in the teachinglearning process. Learning strategies are used when the student shows signs of contimually adjusting to the changes and variations that occur in the course of the activity, always with the ultimate aim of achiering the objective pursued in the most efficient way possible. In this way, the student minimizes the number of errors prior to solving the problem, ensuring that her answer is correct after a minimum of attempts. In addition, autonomous learning is the process that allows the student to regulate what he learns and to be aware of his cognitive and socio-affective processes. The pedagogical effort focuses on the training of individuals with the ability to solve specific aspects of their own learning, that is, guiding the learner to question, review, plan, control and exaluate their own action of learning to learn and print meaningful learning using appropriate and motivating strategies in their learning process.

Keywords: Learning strategies, learning in schoolchildren.

\section{RESUMO}

O objetivo deste estudo foi analisar a formação inicial considerando a exclusão digital em tempos de pandemia na província constitucional de Callao. Foi utilizada uma abordagem transversal mista e um desenho não experimental. Como parte das técnicas de coleta de dados, foram utilizados dois questionários e duas entrevistas. Participaram 377 pais e mães e 334 professores, que faziam parte da comunidade de centros de educação infantil da província constitucional de Callao. 24 pais e 37 professores participaram das entrevistas. Para o processamento das informações foi utilizado o programa Excel. Os resultados indicaram: $41,64 \%$ dos pais não tinham conhecimento de como são utilizadas as ferramentas digitais; e $13,47 \%$ dos professores expressaram o mesmo. Uma das principais conclusões gira em torno do fato de os professores desenvolverem estratégias de apoio ou acompanhamento às familias, reconhecer e aproveitar o papel ativo dos pais na aprendizagem dos filhos. E se comprometeram com o desafio de desenvolver aulas online para meninos e meninas da educação inicial, atuando a partir de sua realidade e adaptando-se aos recursos de que dispunham.

Palavras-chave: Educação inicial; brecha digital; pais de familia; professores. 


\section{Introducción}

Las estrategias de aprendizaje bien establecidas por el docente permiten a los estudiantes un mayor conocimiento, debido a que podemos considerarlas como las herramientas fundamentales para la enseñanza. Fomentando así el desarrollo óptimo de las habilidades cognitivas y meta cognitivas. La enseñanza y el aprendizaje son procesos que se presentan juntos, por lo tanto, las estrategias que se emplean para la instrucción inciden en los aprendizajes.

Maldonado, (2019). Afirma que, los estudiantes deben ser capaces de aprender en forma autónoma y autorregulada. Además, es importante mencionar que, las estrategias de aprendizaje se definen en relación a la toma de decisiones. El estudiante elige y activa aquellos conocimientos que necesita para responder a las exigencias de la demanda profesional y personal, en función de las condiciones de la situación educativa. Por otra parte, la importancia de las estrategias de aprendizaje viene dada por el hecho de que engloban aquellos recursos cognitivos que utiliza el estudiante cuando se enfrenta al aprendizaje. Valle, (1998).

Existen muchos tipos de estrategias de aprendizaje, y se detallará las más importantes: Estrategias de ensayo. Este tipo de estrategia se basa principalmente en la repetición de los contenidos ya sea escrito o hablado. Es una técnica efectiva que permite utilizar la táctica de la repetición como base de recordatorio. Podemos leer en voz alta, copiar material, tomar apuntes. Estrategias de elaboración. Este tipo de estrategia, se basa en crear uniones entre lo nuevo y lo familiar, por ejemplo: resumir, tomar notas libres, responder preguntas, describir como se relaciona la información, buscar sinónimos. Las Estrategias de organización. Se basan en una serie de modos de actuación que consisten en agrupar la información para que sea más sencilla para estudiarla y comprenderla. El aprendizaje en esta estrategia es muy efectivo, porque con las técnicas de: resumir textos, esquemas, subrayado, etc... Podemos incurrir un aprendizaje más duradero, no sólo en la parte de estudio, sino en la parte de la comprensión. La organización deberá ser guiada por el profesor, aunque en última instancia será el estudiante el que se organice. Vásquez, (2017). 
Este artículo de revisión tiene como objetivo revisar las aportaciones más relevantes sobre el tema de las estrategias de aprendizaje de diversos autores e identificar las estrategias pedagógicas que se implementan con mayor frecuencia en la escuela, tradicionales e innovadoras, al mismo tiempo, describir las estrategias de aprendizaje predominantes que se relaciona con el desarrollo de la autonomía de los estudiantes.

Luego de revisar los autores sobre estrategias de aprendizaje, debo mencionar que la mayoría de ellos tienen lógica en sus enunciados, por lo que personalmente pienso que el enseñar estrategias de aprendizaje a los estudiantes debe ser claro y preciso, de tal manera que el estudiante aplique esta técnica en su labor diaria y obtenga buenos resultados en su rendimiento académico, sino fuera así deberá revisar cuidadosamente la estrategia y deberá corregir algún aspecto que probablemente está mal aplicado.

\section{Método}

La metodología utilizada es la siguiente: Búsqueda bibliográfica. - Se revisaron diversos artículos científicos relacionados al tema de Estrategias de Aprendizaje. Criterios de selección. - La selección de los artículos científicos seleccionados fue a través de las páginas web específicas que contienen el tema de investigación. Recuperación de la información. - Las fuentes documentales de donde se recopiló la información son las páginas especializadas como: Scielo, Redalyc, Proquest, Scopus, Google Académico y otras. Evaluación de la calidad de los artículos seleccionados. - La evaluación de los artículos científicos seleccionados fue en base a la similitud con el tema de investigación en estudio. Análisis de la variabilidad y validez de los artículos. - El análisis de la variabilidad y validez de los artículos es a criterio del suscrito dado mi amplio conocimiento sobre el tema que vengo desarrollando.

La metodología didáctica, según Fortea Bagán, (2009), es la estrategia de enseñanza que propone un docente para el trabajo en el aula. Se parte de la metodología, como medio para el logro de los objetivos, y de la didáctica, como herramienta de esa metodología, en procura de los resultados esperados. La didáctica se ha referido a enseñar, instruir, informar, aprender, y toda función dentro del proceso de enseñanza y aprendizaje; en tanto la metodología es el medio del que se sirve la didáctica para transitar. Por lo tanto, se puede decir que se ha hecho uso de la didáctica, cuando el proceso de enseñanza facilita la aplicación de la información o del conocimiento, considerando aspectos como el 
contexto, condiciones e intereses, y las adaptaciones que se hagan a un recurso para centrar la enseñanza en los estudiantes.

Extraer los recursos propios del contexto en que se está inmerso, proporciona mayores beneficios porque es ahí en donde se presentan las necesidades que tienen y desean satisfacer las personas usuarias, y donde esperan encontrar respuesta a los intereses que han centrado su atención, apoyándose de la ambientación metodológica para incentivar el interés en la información de manera creativa y atractiva. Por esta razón, Gómez y García (2014) consideran la didáctica como un proceso de acciones que involucra modelos metodológicos y estratégicos para conseguir el aprendizaje. Orellana, (2017).

Según Pamplona, (2019), la metodología de este artículo está sustentado desde el enfoque cualitativo a partir del método de investigación documental de tipo descriptivo. Este corresponde a un estudio de revisión de la literatura a través de un proceso sistemático de búsqueda, clasificación y análisis de las fuentes de acuerdo con los criterios definidos Booth, Papaioannou \& Sutto, (2012), la cual se establece la búsqueda, selección y análisis rigurosa, como resultados de investigaciones científicas de los últimos años de las cuales se realizó un proceso de selección exhaustivo eligiendo solo artículos de fuentes primarias (originales).

\section{Desarrollo y discusión}

El objetivo principal fue establecer la estrategia de aprendizaje predominante que se relacione con el desarrollo de la autonomía de los estudiantes. Para lograr en los estudiantes un nivel significativo de comprensión del manejo de información se debe considerar las estrategias de aprendizaje pertinentes y adecuadas a la realidad estudiantil.

En ese sentido, es una exigencia social que los estudiantes adquieran capacidades de aprendizaje que les permita adaptarse mejor al nivel superior y las nuevas demandas laborales. Deben ser capaces de aprender en forma autónoma y autorregulada. Es importante mencionar que, las estrategias de aprendizaje se definen en relación a la toma de decisiones. Maldonado, (2019).

Başbaği y Yilmaz, (2015). Afirman que tanto los estudiantes de secundaria como los de universidad dominan un sinfín de estrategias de aprendizaje, pero se les complica emplearlas. Aplicar una estrategia a una actividad específica de aprendizaje es uno de los 
problemas más grandes. La estrategia de codificación de información ejerce mayor peso en el aprendizaje autónomo en los estudiantes. En oposición a ello, Marrugán, (2013) concluyó que la inteligencia general y estrategias de recuperación de la información no muestran relaciones significativas.

La estrategia de adquisición de información denominado exploración es la de mayor peso sobre el aprendizaje autónomo. En respaldo a ello, Tobón, (2012), demostró que la falta de utilización de estrategias pedagógicas no conduce y no mejora el desarrollo del pensamiento crítico. Asimismo, Loret de Mola, (2011), en el estudio "Estilos y estrategias de aprendizaje en el rendimiento académico de los estudiantes de la Universidad Peruana Los Andes de Huancayo" demostró que, a mejor estilo y mejor uso de estrategias, el rendimiento académico será óptimo.

Según Vásquez, (2017). Indica que los estudiantes del 2do de secundaria adquieren conocimientos necesarios y óptimos para su aprendizaje mediante las estrategias didácticas que emplean los docentes, por otro lado, los estudiantes emplean sabiamente estrategias de aprendizaje que favorecen el desarrollo de las habilidades cognitivas de los mismos.

Según Visbal, (2017). Menciona que de acuerdo con Porter, citado por Martínez y

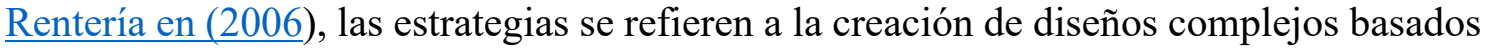
en el análisis que brinda una posición valiosa y original, articulada con toda una estructura de acciones y comportamientos dirigidos hacia un objetivo determinado. El aprendizaje implica, además, la creación de nexos entre lo mental, lo socio afectivo, lo sensomotriz y lo neurológico, lo cual afecta factores personales, relacionales, cognitivos y simbólicos ligados a cambios, o reorganizaciones. Tradicionalmente, las estrategias de aprendizaje se han conceptualizado como una combinación de procesos cognitivos y meta cognitivos, Peculea y Bocos, (2015).

Por otra parte, Roux, (2015). Sostiene que las estrategias de aprendizaje son actividades o procesos mentales que llevan a cabo los y las estudiantes intencionalmente para procesar, entender y adoptar la información que reciben en el proceso educativo. Este artículo presenta un estudio correlacional que examinó el uso de estrategias de aprendizaje de 162 estudiantes de educación media superior en un colegio privado del 
noreste de México. Los objetivos eran identificar las estrategias de aprendizaje más utilizadas y conocer la relación entre las estrategias empleadas y las calificaciones reportadas para el período escolar inmediato anterior a la realización del estudio. Se usó el Cuestionario de Evaluación de las Estrategias de Aprendizaje para Estudiantes Universitarios (CEVEAPEU).

Las estrategias más utilizadas fueron las motivacionales (ejemplo: la inteligencia se puede incrementar con el esfuerzo) y las Meta cognitivas (ejemplo: procuro aprender de mis errores). La estrategia que mostró mayor correlación con el rendimiento académico fue: tomar apuntes en clase, que es una estrategia de procesamiento y uso de información. Los reactivos que resultaron con baja correlación fueron: la inteligencia se tiene o no y no se tiene y no se puede mejorar (motivacional), y aporto ideas personales (procesamiento y uso de información). El estudio sugiere adaptar el instrumento para su uso en el nivel medio superior y poner mayor atención a la enseñanza de las estrategias de aprendizaje en la educación media superior mexicano. Sobre todo, las que se usan en la selección, procesamiento y uso de la información, necesarias en el desarrollo de una sociedad basada en el conocimiento.

Para Marín, (2008). La investigación básica "Se caracteriza porque parte de un marco teórico y permanece en él; el objetivo reside en formular nuevas teorías o modificar las que ya existen, en desarrollar los conocimientos científicos o filosóficos, pero sin contrastarlos con ningún aspecto práctico". Mediante esta investigación llegamos a la conclusión, que los docentes consideran de vital importancia el uso adecuado de las estrategias didácticas y que a través de ellos permite mejorar las habilidades cognitivas de sus estudiantes.

El estudiante desarrolla una capacidad crítica y busca propósitos hacia los hechos sociales y desarrollar habilidades y destrezas, para lograr participar de manera activa en su entorno social.

En base a los comentarios anteriores, y a modo de síntesis y delimitación conceptual, los rasgos característicos más destacados de las estrategias de aprendizaje podrían ser los siguientes Pozo y Postigo, (1993): 
a. Su aplicación no es automática sino controlada. Precisan planificación y control de la ejecución y están relacionadas con la meta cognición o conocimiento sobre los propios procesos mentales.

b. Implican un uso selectivo de los propios recursos y capacidades disponibles. Para que un estudiante pueda poner en marcha una estrategia debe disponer de recursos alternativos, entre los que decide utilizar, en función de las demandas de la tarea, aquellos que él cree más adecuados.

c. Las estrategias están constituidas de otros elementos más simples, que son las técnicas o tácticas de aprendizaje y las destrezas o habilidades. De hecho, el uso eficaz de una estrategia depende en buena medida de las técnicas que la componen. En todo caso, el dominio de las estrategias de aprendizaje requiere, además de destreza en el dominio de ciertas técnicas, una reflexión profunda sobre el modo de utilizarlas o, en otras palabras, un uso reflexivo y no sólo mecánico o automático de las mismas. Pozo, (1989).

\section{¿Cuáles son las estrategias de aprendizaje?}

Las estrategias de aprendizaje se clasifican de la siguiente manera, según Román y Gallego, (1994).

1. Escala de estrategias de adquisición de información. Adquisición de información es atender porque los procesos atencionales, son los que se encargan de seleccionar, transformar y transportar la información desde el ambiente al registro sensorial. Primordial para captar o adquirir información es atender; una vez atendida, lo más probable es que se pongan en marcha procesos de repetición, encargados de llevar la información transportando y transformando, junto a los atencionales y en interacción con ellos, desde el registro sensorial a la memoria de corto plazo y de aquí se selecciona la información procedente, a la memoria de largo plazo. Las estrategias de atención son aquellos que favorecen el control o dirección de todo el sistema cognitivo hacia la información relevante de cada contexto; por otra parte, captar una información es percibir o darse cuenta de la información que se recibe, que pueden llegar por vía oral, escrita o por percepción del ambiente. Bernardo, (2007). 
De la misma forma este investigador describe que dentro de las estrategias de adquisición de información hay dos tipos de estrategias:

a) Estrategias atencionales. Estas estrategias son: subrayado lineal, cuya finalidad es destacar lo que se considera importante en un texto, mediante el rayado en la parte inferior de palabras o frases; subrayado idiosincrático, es destacar lo que se considera importante en un texto mediante la utilización de signos, colores y formas propios de quien los utiliza; epigrafiado, es distinguir partes, puntos importantes o cuerpos de conocimientos en un texto mediante anotaciones, títulos o epígrafes.

b) Estrategias de repetición. Tienen la función de hacer durar o hacer lo posible y facilita el paso de la información a la memoria de largo plazo. Estas estrategias están integradas por los siguientes: repaso en voz alta, que ayuda a la memorización pronunciando las palabras fuertes debido a que intervienen dos sentidos, la vista y el oído; repaso mental, es reflexionar sobre lo leído o estudiado y sacar el resumen mentalmente; y el repaso reiterado, es leer varias veces el tema con pequeñas pausas para reflexionar sobre lo comprendido.

2. Escala de estrategias de codificación de información. El paso de la información de memoria de corto plazo a la memoria de largo plazo requiere, de los procesos de atención y de repetición. Codificar es traducir a un código. Las estrategias de codificación se clasifican en: a) Estrategias de memotecnización; significa técnicas de memorización para recordar secuencias; la mnemotecnia ofrece artificios para salvar estas dificultades.

Es transformar información a memorizar poco conocida en información conocida. Bernardo, (2007). b) Estrategias de elaboración, según, Weinstein y Mayer citado por Román y Gallego, (1994), distinguen dos niveles de elaboración: el simple, basado en la asociación intra material a aprender, y el complejo, que lleva a cabo la integración en los conocimientos previos del individuo. El almacenamiento duradero parece depender más de la elaboración y/o organización de la información que da las nemotecnias. c) Estrategias de organización. Hacen que la información sea más significativa y manejable (concreto para el estudiante). La organización de información previamente elaborada tiene lugar según las características del estudiante, de acuerdo a sus capacidades. 
Las estrategias de organización se pueden clasificar de diferentes formas: mediante agrupamiento diversos como resúmenes y esquemas; secuencias lógicas, como: causa efecto, problema- solución, comparación, etc.; construyendo mapas conceptuales de Novack, mapeo de Armbruster y Anderson, reticulación de Dansereau; y diseñando diagramas como matrices, cartesianas, diagramas de flujo, diagramas en $\mathrm{V}$, entre otras. Es recomendable iconografíar para que exista relación, significatividad y estilo en los mapas Novak, (1998).

3. Escala de estrategias de recuperación de información. Son los que favorecen la búsqueda de información en la memoria y generación de respuesta. El sistema cognitivo cuenta con la capacidad de recuperación o de recuerdo del conocimiento almacenado en la memoria de largo plazo; esta escala identifica y evalúa en qué medida las estrategias de recuperación favorece la búsqueda de información en la memoria y la generación de respuesta mediante sistemas de búsqueda y/o generación de respuesta.

Entre las estrategias de recuperación que se tiene: a) Estrategias de búsqueda; las estrategias para la búsqueda de la información almacenada se hallan básicamente condicionadas por la organización de los conocimientos en la memoria, resultado a su vez de las estrategias de codificación. La calidad de los esquemas, o sea estructuras abstractas de conocimientos, elaborados constituyen el campo de búsqueda. Por tanto, las tácticas de búsqueda que tienen lugar en un individuo guardan correspondencia con los utilizados por el mismo para la codificación.

Además, las estrategias de búsqueda sirven para facilitar el control o la dirección de búsqueda de palabras, significados y representaciones conceptuales o icónicas en la memoria de largo plazo. (b) Búsqueda de indicios, es decir, permite conocer la existencia de otro no percibido. c) Estrategias de generación de respuestas; la generación de una respuesta debidamente realizada puede garantizar la adaptación positiva que se deriva de una conducta adecuada a la situación.

4. Escala de estrategias de apoyo al procesamiento. Las estrategias de apoyo, apoyan, ayudan y potencian el rendimiento de las estrategias de adquisición, de codificación y de recuperación incrementando la motivación, la autoestima, la atención. 
Garantizan el clima adecuado para un buen funcionamiento de todo el sistema cognitivo, hay tres tipos de estrategias de apoyo: un control meta cognitivo, que conduce al estudiante lúcidamente desde el principio hasta el fin de su proceso de aprendizaje; un control de procesos afectivos como las ansiedades, las expectativas, la atención; y un control de procesos sociales como la habilidad para obtener apoyo, evitar conflicto, cooperar, competir, motivar a otros, etc. Sin embargo, Román y Gallego, (1994), consideran en las estrategias de apoyo a estrategias meta cognitivas y estrategias socio afectivas.

a) Estrategias meta cognitivas: están relacionadas con el conocimiento que el estudiante tiene de sus propios procesos de su conocimiento y con el control de esos mismos procesos. Tejedor y García-Valcárcel, (1996). b) Estrategias socio afectivas: se relacionan con el interés y motivación para el aprendizaje. García, (2002); estas estrategias se pueden dividir en: estrategias sociales, que facilitan el aprendizaje en interacción con los demás y ayudan a los aprendices a entender mejor la cultura a la que se enfrentan; estrategias afectivas, son las capacidades para identificar los propios sentimientos y ser consciente de las circunstancias que evocan el proceso de enseñanza aprendizaje. Cabañas, (2008).

En forma similar, Freiberg, (2017), sostiene que los estilos y las estrategias de aprendizaje describen las preferencias de los estudiantes durante las actividades de aprendizaje que involucran el incorporar y conciliar exitosamente información novedosa con la preexistente.

Por su parte Maldonado, (2019), menciona que la estrategia de codificación de información ejerce mayor peso en el desarrollo de la autonomía de los estudiantes. Así también, es una estrategia de riesgo que significa que un estudiante que no la maneje presentará siempre bajos niveles de aprendizaje autónomo.

Lozano, (2018). Presenta un trabajo teórico sobre los antecedentes históricos de la evolución del estudio de las dificultades de aprendizaje. Se explican diferentes teorías que han tenido una aportación significativa en el tratamiento de dichas dificultades y también cómo los estudiantes con dificultades específicas de aprendizaje (DEA) han sido tratados en el entorno escolar a lo largo de la historia. Se comentan factores socio-educativos que pueden interferir en los procesos de enseñanza / aprendizaje. Se han estudiado las nuevas 
teorías más relevantes en el desarrollo de la inteligencia y sus consecuencias para el estudio de las DEA. Se observa cómo la fluidez lectora es uno de los componentes necesarios para ejecutar una buena lectura y también la importante relación de ésta con la comprensión y eficacia lectora.

García, (2015). Expone el resultado de dos investigaciones desarrolladas con el propósito de determinar el repertorio de estrategias de aprendizaje (EA) y su relación con el avance curricular y el rendimiento académico de la población estudiantil de dos instituciones de Educación Superior Chilenas, ubicadas en la V y VIII región del país. En ambos casos se aplicó el test de Adquisición, Codificación, Recuperación y Apoyo al Procesamiento de la Información (ACRA). El primer caso corresponde a jóvenes de una carrera de formación de docentes en Educación Primaria y el segundo, a estudiantes pertenecientes a una carrera de nivel técnico, de una institución de las Fuerzas Armadas chilenas. Los hallazgos del estudio develaron que, en general, ambos grupos al ser medidos, contaban con un repertorio de EA escasas. Por otra parte, en el supuesto de que las EA aumentaban mientras se avanzaba en la formación curricular, las evidencias arrojaron que ello no ocurre y que estas, se mantienen estables a lo largo de dicho proceso. Finalmente, se corroboró lo que también, en otros trabajos de investigación se ha descubierto, en cuanto a que los estudiantes que tienen un buen rendimiento poseen un repertorio mayor de EA que los que logran un menor rendimiento.

Pamplona, (2019). Da a conocer los principales hallazgos de investigaciones que describen las estrategias de enseñanza implementadas por el docente en educación primaria para el aprendizaje en las áreas básicas. Se concluye que es necesario que los docentes hagan divulgación científica de sus prácticas educativas para que otros docentes repliquen estos recursos y se actualicen continuamente para fortalecer sus procesos de enseñanza aprendizaje y logren el desarrollo de competencias en sus estudiantes a través de estrategias metodológicas pertinentes.

Cortes, (2017). Orientó su investigación al análisis de las estrategias de enseñanza utilizadas por los docentes del grado $4^{\circ}$ en el aprendizaje de estudiantes considerados con bajo rendimiento académico del grado cuarto de una institución educativa del barrio popular número dos de la ciudad de Medellín. Los resultados del estudio muestran que las estrategias de enseñanza de los docentes se caracterizan por ser de tipo expositivas y 
evaluativas las cuales inciden en procesos de atención y memorización en los estudiantes con bajo rendimiento académico. Además, las estrategias de evaluación pueden influir mucho más, porque se realiza una valoración alta de las tareas y evaluaciones programadas para las notas definitivas del periodo, más que del desempeño demostrado por el estudiante en el proceso de las clases.

Alvarado, (2019). Una de las aportaciones del análisis de las trayectorias en la educación superior es permitir identificar tiempos, eficiencia y rendimiento escolares para implementar acciones y mejorar la calidad de los servicios educativos. Los resultados mostraron algunas diferencias con datos favorables para el que alcanzó el 100\% de créditos tanto en el desempeño escolar como en la utilización de estrategias de aprendizaje, sin embargo, se requiere continuar la investigación para determinar la relación entre ambas variables.

Segura, (2018). Menciona que la función formativa, cuyo propósito es regular el proceso educativo en el aula, es decir, el trabajo escolar cotidiano, el cual se integra a la intervención didáctica ya que está centrada en los procesos más que en los resultados de aprendizaje, por lo que la realimentación-pro alimentación que se obtiene es de carácter cualitativo. Ello permite detectar las barreras de aprendizaje para el desarrollo de la mediación pedagógica del estudiantado. La función formativa conlleva a tomar en cuenta la diversidad desde procesos como la realimentación, pro alimentación, la sostenibilidad de la evaluación y las barreras para el aprendizaje. Se concluye que el personal docente observado y posteriormente entrevistado aún no logra tener claridad sobre la forma en que se califica el componente de trabajo cotidiano.

Para Monereo, (1994). Las estrategias de aprendizaje son procesos de toma de decisiones (conscientes e intencionales) en los cuales el estudiante elige y recupera, de manera coordinada, los conocimientos que necesita para cumplimentar una determinada demanda u objetivo, dependiendo de las características de la situación educativa en que se produce la acción. Por su parte para Schmeck (1988) y Schunk (1991), afirman que las estrategias de aprendizaje son secuencias de procedimientos o planes orientados hacia la consecución de metas de aprendizaje, mientras que los procedimientos específicos dentro de esa secuencia se denominan tácticas de aprendizaje. 
En este caso, las estrategias serían procedimientos de nivel superior que incluirían diferentes tácticas o técnicas de aprendizaje. Asimismo, los estudios sobre estrategias de aprendizaje pueden considerarse una de las líneas de investigación más fructíferas a lo largo de los últimos años dentro del ámbito del aprendizaje escolar y de los factores que inciden en el mismo. Para Entwistle y Marton, (1991), las investigaciones sobre las estrategias de aprendizaje junto con la teoría del procesamiento de la información constituyen las aportaciones más relevantes de la psicología cognitiva al estudio del aprendizaje escolar.

En consecuencia, podemos decir que las estrategias de aprendizaje constituyen actividades conscientes e intencionales que guían las acciones a seguir para alcanzar determinadas metas de aprendizaje. Con ciertos matices, quizás sea más exacto afirmar que son actividades potencialmente conscientes y controlables, Pressley, ForrestPressley, Elliott-Faust y Miller, (1985); aunque debemos subrayar, siguiendo a Beltrán (1993), que un rasgo importante de cualquier estrategia es que está bajo el control del estudiante, es decir, a pesar de que ciertas rutinas pueden ser aprendidas hasta el punto de automatizarse, las estrategias son generalmente deliberadas, planificadas $\mathrm{y}$ conscientemente comprometidas en actividades. Dicho, en otros términos, las estrategias de aprendizaje son procedimientos que se aplican de un modo intencional y deliberado a una tarea y que no pueden reducirse a rutinas automatizadas. Selmes, (1988), es decir, son más que simples secuencias o aglomeraciones de habilidades. Nisbet y Shucksmith, (1987).

Según Valle, (1998), cuando el estudiante se enfrenta a la resolución de una determinada tarea dispone de una amplia variedad de recursos mentales que pueden contribuir a una solución adecuada de la misma. No obstante, los posibles motivos, intenciones y metas del sujeto son elementos que van a condicionar en gran medida el tipo de estrategias que va a utilizar para resolver dicha tarea. En este caso, las propias creencias del sujeto respecto a su capacidad para enfrentarse a la resolución de la tarea, así como la importancia e interés de la misma, son algunos de los factores motivacionales que pueden determinar la puesta en marcha de unas determinadas estrategias.

Klimenko, (2009). Sostiene que, una de las prioridades de la enseñanza en la escuela contemporánea es ayudar a los estudiantes a convertirse en agentes autónomos que 
gestionen su aprendizaje. El papel que debe cumplir el profesor para apoyar el aprendizaje es el de mediador y orientador, a fin de proporcionar a los estudiantes herramientas necesarias para que aprendan a organizar y dirigir sus propios procesos y actividades de estudio. En este orden de ideas, la enseñanza de estrategias cognitivas y meta cognitivas es una alternativa para mejorar los procesos de aprendizaje, sobre todo en los alumnos con dificultades en este aspecto, como, por ejemplo, niños afectados por problemas atencionales.

\section{Conclusión}

Todas las estrategias de aprendizaje son importantes y complementarias, permiten desde diferentes técnicas que se aprenda el conocimiento recibido y pueda ser aplicado y manejado con facilidad por la persona que las utilice. De acuerdo al tipo de conocimiento es preciso utilizar alguna técnica para el aprendizaje que mejora la receptibilidad hacia el mismo y, por consiguiente, contribuye a su mejor aprovechamiento.

Los artículos revisados nos permitieron identificar algunas estrategias de aprendizaje que influyen en el rendimiento académico de estudiantes de pregrado con lo cual las instituciones educativas podrían desarrollar programas de intervención para potenciar las mismas. Se destaca lo siguiente: que las mujeres tienen una mayor probabilidad de tener un rendimiento académico bueno en comparación de los hombres y el estrato social o el tipo de institución educativa no tienen una influencia significativa en el rendimiento académico de los estudiantes. Visbal, (2017).

Asimismo, León y otros, (2015), mencionan que, en cuanto a las estrategias de organización, cuando los estudiantes realizan un nuevo tema, no todos emplean los organizadores para abordar los nuevos conocimientos. Es necesario que los profesores utilicen los diferentes organizadores en el desarrollo de sus clases -los mapas mentales y conceptuales, los mentefactos conceptuales y proposicionales, el árbol comparativo, los cuadros sinópticos u otros-, para que los estudiantes aprendan a encontrar relaciones, estructuren el conocimiento, lo relacionen con lo que ya saben y le encuentren nuevos significados, mismos que recordarán porque los esquemas que se realicen tendrán cierta lógica que no se olvida. Además, los pueden utilizar para abordar cualquier temática teórica o práctica. 
Las estrategias de codificación de información ejercen mayor peso en el desarrollo de la autonomía de los estudiantes. Así también, son estrategias de riesgo que significa que un estudiante que no la maneje presentará siempre bajos niveles de aprendizaje autónomo. Maldonado, (2019).

Cuando los estudiantes se enfrentan en el abordaje del aprendizaje de un tema nuevo, las estrategias de apoyo al procesamiento: meta cognitiva de planeación - como el repaso de los temas anteriores, la programación de horarios, el fijarse metas y buscar los recursos necesarios- favorecen los procesos educativos por competencias, aspectos que permiten la autonomía, la independencia y la autorregulación para “aprender a aprender".

Las estrategias de adquisición de la información son fuentes de información para indagar en los temas que se estudian como el internet, los libros y las preguntas al profesor favorecen la autorregulación de los aprendizajes.

Asimismo, las estrategias de recuperación de información ayudan al estudiante a realizar el ejercicio constante y permanente de las actividades de evaluación con el auto apoyo afectivo, de esta forma podrán reconocer sus alcances y limitaciones, aspectos que permitirán buscar las estrategias oportunas para aclarar sus dudas; así lograrán forjar su autoformación para conseguir la responsabilidad y la autonomía necesarias para alcanzar el éxito de los aprendizajes significativos.

Todas las estrategias de aprendizaje tienen que tener relación con el modelo educativo por competencias y garantizar espacios para reconocer el interés y la motivación de los estudiantes, adquiriendo la formación y el aprendizaje que les permitirán desempeñarse en su vida laboral. De esta manera, lograrán adquirir el equilibrio emocional necesario y aprenderán a autorregularse para el logro de competencias cognitivas y actitudinales autónomas, adoptando la responsabilidad de su propio aprendizaje, sin sentirse afectados emotivamente. León, (2016). 


\section{Figura 1}

Mapa Mental De Las Estrategias De Aprendizaje
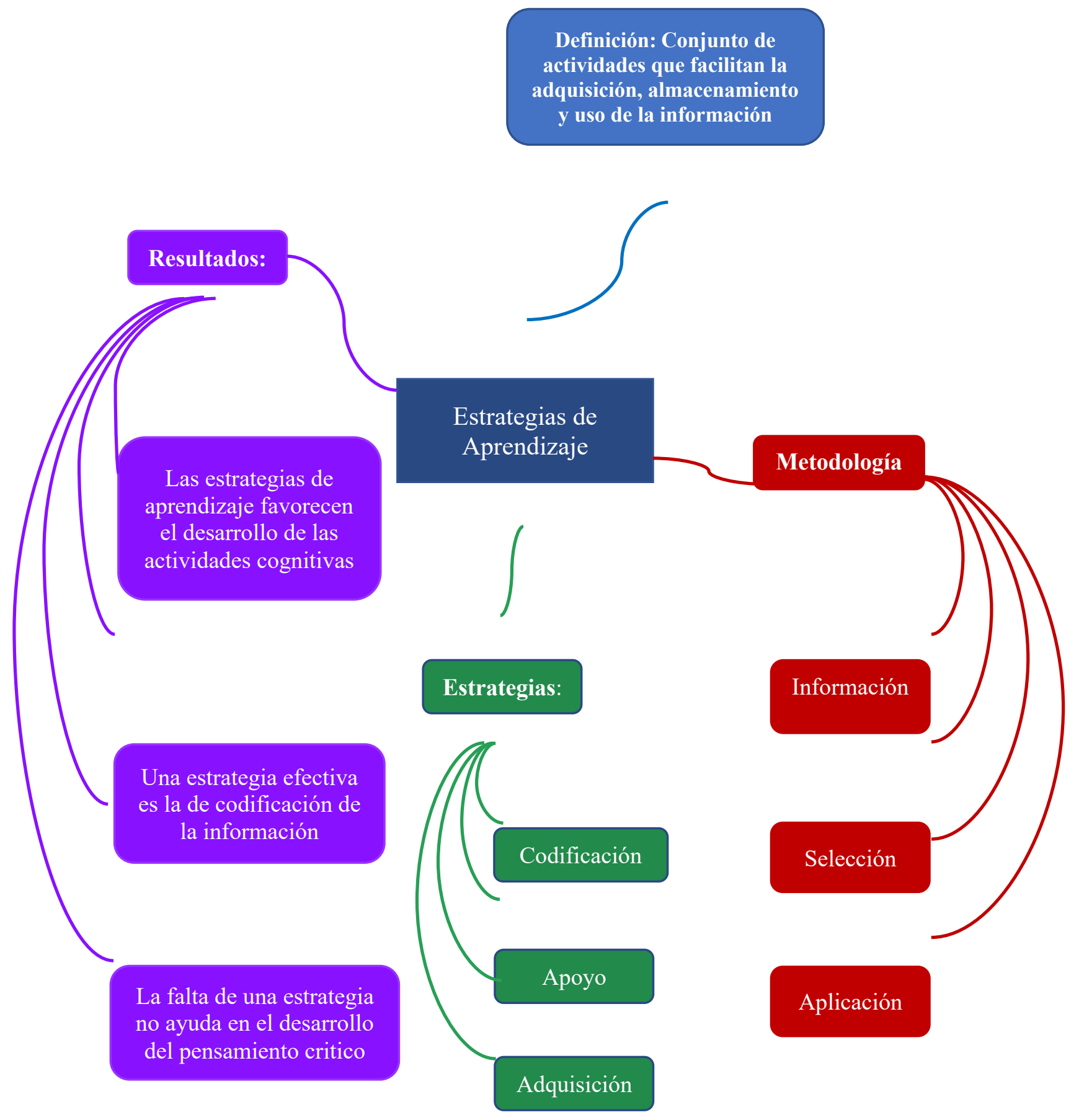

Evaluación

Las estrategias de aprendizaje

Recuperación requieren una reflexión profunda sobre el modo de utilizarlas

NOTA: La figura 1 muestra el proceso de investigación logrado de los artículos revisados sobre las estrategias de aprendizaje. 
Referencias

Alvarado, G. (2019). “Desempeño escolar y estrategias de aprendizaje en estudiantes universitarios con trayectoria escolar adecuada”. Rev Elec Psic Izt. 2019.

Başbaği, L., \& Yilmaz, O. (2015). Autonomous foreing language learning. Journal of Languages and Culture, 6 (8), 71-79. Doi: https://doi.org/10.5897/JLC2015.0338

Beltrán, J. (1993). Procesos, estrategias y técnicas de aprendizaje. Madrid:

Síntesis.

Cortes, A. (2017). “Estrategias pedagógicas que favorecen el aprendizaje de niñas y niños de 0 a 6 años de edad en Villavicencio-Colombia”. Disponible en: https://revistas.usantotomas.edu.co/index.php/riiep/article/download/4746/4484

Cortes, C. (2017). "Estrategias de enseñanza y aprendizaje en estudiantes con bajo rendimiento académico de $4^{\circ}$ ". Recuperado de: https://dspace.tdea.edu.co/ bitstream/handle/tda/330/estrategias $\% 20$ de $\% 20$ ensenanza $\% 20 \mathrm{y} \% 20$ aprendizaje $\% 20$ en \%20estudiantes $\% 20$ con $\% 20$ bajo $\% 20$ rendimiento.pdf;jsessionid=203cf211bacaeb8df67 $\underline{89 \mathrm{f} 74 \mathrm{ede} 6 \mathrm{~b} 546 ? \text { sequence }=4}$

Entwistle, N. J. y Marton, F. (1991). Knowledge objects: Understandings constituted through intensive academic study. Bristish Journal of Educational Psychology.

Freiberg, A. (2017). “Estilos y estrategias de aprendizaje en estudiantes universitarios de Buenos Aires”. Revista de Psicología vol.35 no.2 Lima jul./dic. 2017

García, F. (2015). “Aprendizaje y rendimiento académico en educación superior: un estudio comparado". Revista Electrónica "Actualidades Investigativas en Educación", vol. 15, núm. 3, septiembre-diciembre, 2015, Universidad de Costa Rica.

García, J. (2015). “Las estrategias utilizadas por los niños tee savi en la resolución de problemas aritméticos”. Revista Latinoamericana de Investigación en Matemática Educativa, vol. 18, núm. 2, 2015. 
Klimenko. O. (2009). La enseñanza de las estrategias cognitivas y meta cognitivas como una vía de apoyo para el aprendizaje autónomo en los niños con déficit de atención sostenida. "Revista Virtual Universidad Católica del Norte". No.27. Universidad de Antioquia, Medellín. Colombia.

León A., Risco, E., y Alarcón, C. (2016). estrategias de aprendizaje en Educación Superior en un modelo curricular de competencias. Revista de la Educación Superior. Vol. 43. Núm. 172. Recuperado de: https://www.elsevier.es/es-revista-revista-educacionsuperior-216-articulo-estrategias-aprendizaje-educacion-superior-un$\underline{\mathrm{S} 0185276015000552}$

Loret de Mola. (2011). Estilos y estrategias de aprendizaje en el rendimiento académico de los estudiantes de la Universidad Peruana los Andes, Revista estilos de aprendizaje, 4(8), 149- $\quad 184 . \quad$ Recuperado de: http://learningstyles.uvu.edu/index.php/jls/article/view/69/45

Lozano, J. (2018). “Las dificultades de aprendizaje en los centros educativos de enseñanza secundaria”. Programa de intervención en la fluidez y comprensión lectora. Recuperado de: https://eprints.ucm.es/55409/1/T41123.pdf

Maldonado, M. (2019) “Estrategias de aprendizaje para el desarrollo de la autonomía de los estudiantes de secundaria”. Propós. Represente. vol.7 no.2 Lima mayo/agos. 2019

Martínez, Á, \& Rentería Pérez, E. (2006). Estrategias de aprendizaje para la empleabilidad en el mercado del trabajo de profesionales recién egresados. Universitas psychologica, Vol. 6, No. 1, 89-104.

Monereo. C. (Coord.) (1994). Estrategias de enseñanza y aprendizaje. Formación del profesorado y aplicación en la escuela. Barcelona: Graó.

Nisbet, J. y Shucksmith, J. (1987). Estrategias de aprendizaje. Madrid: Santillana, 1987. 
Orellana, C. (2017). “La estrategia didáctica y su uso dentro del proceso de enseñanza y aprendizaje en el contexto de las bibliotecas escolares”. VOLUMEN 7, NÚMERO 1: ENERO-JUNIO 2017. DOI 10.15517/ECI.V7I1.27241

Pamplona, J., Cuesta, J.C. y Cano, V. (2019). “Estrategias de enseñanza del docente en las áreas básicas: una mirada al aprendizaje escolar”. Revista Eleuthera, 21, 13-33. DOI: 10.17151/eleu.2019.21.2.

Peculea, L., \& Bocos, M. (2015). The Role of Learning Strategies in the Development of the Learning-to-learn Competency of 11 th Graders from Technical Schools. Procedia-Social and Behavioral Sciences, 203, 16-21.

Pozo, J. I. y Postigo, Y. (1993). Las estrategias de aprendizaje como contenido del currículo. En C. Monereo (Compil.), Las estrategias de aprendizaje: Procesos, contenidos e interacción. Barcelona: Domènech.

Pressley, M., Borkowski, J. G. y Schneider, W. (1985). Cognitive strategies: Good strategy users coordinate metacognition and knowledge. Revista Vasta (Ed.), Annals of child development. Vol. 4. Greenwich, Conn.: JAI Press.

Román J. y Gallego S. (1994). ACRA Escalas de estrategias de aprendizaje. Madrid: TEA Ediciones S. A.

Roux, R. (2015). “Estrategias de aprendizaje y su relación con el rendimiento académico en estudiantes de una escuela privada de educación media superior”. Rev. Actual. Investig. Educ vol.15 n.1 San José Jan. /Apr. 2015

Selmes, I. (1988). La mejora de las habilidades para el estudio. Barcelona: Paidós/MEC. (Edic. original: 1987).

Schmeck, R. R. (1988). An introduction to strategies and styles of learning. En R. R. Schmeck (Ed.), Learning strategies and learning styles. New York: Plenum Press. 
Schunk, D. H. (1991). Learning theories. An educational perspective. New York: McMillan.

Segura, M. (2018). “La función formativa de la evaluación en el trabajo escolar cotidiano”. Revista Educación, vol. 42, núm. 1, 2018 Universidad de Costa Rica, Costa Rica.

Valle, A., González R., Cuevas L., Fernández, A., (1998). Las estrategias de aprendizaje: características básicas y su relevancia en el contexto escolar. Revista de Psicodidáctica, núm. 6, 1998, Universidad del País Vasco/Euskal Herriko Unibertsitatea Vitoria-Gazteis, España.

Vásquez, M. (2017). “Estrategias de aprendizaje y el desarrollo de habilidades cognitivas de los alumnos del 2 do año de secundaria de la i.e. $n^{\circ} 64237$ “cerfa” distrito de contamana - provincia de Ucayali 2017”. Recuperado de:

http://repositorio.unjfsc.edu.pe/bitstream/handle/UNJFSC/3754/TESIS\%20VASQUEZ \%20PISCO $\% 20$ MANUEL.pdf?sequence=1\&isAllowed=y

Visbal, D. (2017). “Estrategias de aprendizaje en la educación superior”. Sophia, vol. 13, núm. 2, 2017, Universidad La Gran Colombia. Colombia. 